\title{
GEIDST
}

\section{RESISTÊNCIA AOS ANTIBIÓTICOS EM NEISSERIA GONORRHOEAE - PASSADO, PRESENTE E FUTURO}

\author{
Ermelindo Tavares', Cândida Fernandes², Maria José Borrego ${ }^{3}$, Ana Rodrigues², Jorge Cardoso 4 \\ IInterno(a) do Internato Complementar de Dermatologia e Venereologia/Resident Dermatology and Venereology, \\ Hospital Distrital de Santarém EPE, Santarém \\ ${ }^{2}$ Assistente Graduada de Dermatologia e Venereologia/Graduated Consultant of Dermatology and Venereology, \\ Consulta de Doenças Sexualmente Transmissíveis/STDs Clinic, Serviço de Dermatologia e Venereologia, Hospital de \\ Curry Cabral, Centro Hospitalar de Lisboa Central, EPE, Lisboa \\ ${ }^{3}$ Coordenadora do Laboratório Nacional de Referência das Infeções Sexualmente Transmissíveis/Coordinator of the \\ National Laboratory of Sexual Transmitted Infections, Instituto Nacional de Saúde Dr. Ricardo Jorge, Lisboa \\ ${ }^{4}$ Diretor do Serviço de Dermatologia e Venereologia/Director of the Dermatology and Venereology Department, \\ Hospital de Curry Cabral, Centro Hospitalar de Lisboa Central, EPE, Lisboa, Portugal
}

RESUMO - Introdução: A resistência aos antibióticos em Neisseria gonorrhoeae tem-se revelado um importante problema de saúde pública mundial, estando a levantar grandes dificuldades em termos de opções terapêuticas em alguns países. Objectivo: Rever o panorama (nacional e internacional) da resistência aos antibióticos em Neisseria gonorrhoeae. Material e Métodos: Pesquisa de artigos em revistas nacionais e internacionais (estas últimas com indexação na Pubmed/Medline e redigidas em inglês). Utilizaram-se como palavras-chave: "Neisseria gonorrhoeae antibiotic resistance". Resultados: As cefalosporinas de terceira geração (ceftriaxone e cefixima), associadas ou não à azitromicina, substituíram as fluoroquinolonas como fármacos de primeira linha no tratamento da gonorreia. Os relatos de resistência às cefalosporinas são ainda relativamente escassos; contudo, o aumento da concentração inibitória mínima (CIM), traduzido pela diminuição da sensibilidade a esta classe de antibióticos, tem vindo a ser regularmente descrita. Por outro lado, a resistência à azitromicina foi relatada em vários países. Em Portugal, já foram reportados casos de Neisseria gonorrhoeae resistentes à azitromicina mas não às cefalosporinas. Porém, estirpes com diminuição da sensibilidade às cefalosporinas foram já detetadas. Conclusão: Novas alternativas terapêuticas são indispensáveis para o tratamento das infeções por Neisseria gonorrhoeae, bem como condutas adequadas por parte dos médicos e dos doentes e seus contactantes.

PALAVRAS-CHAVE - Antibióticos; Resistência antibiótica; Neisseria gonorrhoeae; Guideline.

\section{ANTIBIOTIC RESISTANCE IN NEISSERIA GONORRHOEAE - PAST, PRESENT AND FUTURE}

ABSTRACT - Introduction: The antibiotic resistance in Neisseria gonorrhoeae has been considered an important public health problem worldwide, posing serious problems for its treatment in some countries. Aims: To overview the national and international antibiotic resistance reports on Neisseria gonorrhoeae. Material and Methods: Literature search in national and international scientific journals (the later indexed in PubMed/Medline and in English language). "Neisseria gonorrhoeae antibiotic resistance" were used as keywords. Results: Third-generation cephalosporins (ceftriaxone and cefixime), alone or combined with azithromycin, replaced fluroquinolones as the first-line treatment for gonorrhea. Up to now, there were just a few reports on cephalosporin resistance. However, reports of an increase of the minimum inhibitory concentration (MIC) to cephalosporins, reflected by the decreased sensitivity to this class of antibiotics, became frequent. The azithromycin resistance has been reported in several countries. In Portugal, to date, there are no described cases of cephalosporin resistance; however, azithromycin - resistant Neisseria gonorrhoeae isolates and reduced sensitivity to cephalosporins have already been described. Conclusion: New therapeutic agents are essential to 
overcome antibiotic resistance in Neisseria gonorrhoeae, as well as appropriate conducts by physicians, patients and sexual contacts of the later.

KEY-WORDS - Anti-Bacterial agents; Drug resistance, bacterial; Neisseria gonorrhoeae; Guideline.

\author{
Conflitos de interesse: Os autores declaram não possuir conflitos de interesse. \\ No conflicts of interest. \\ Suporte financeiro: O presente trabalho não foi suportado por nenhum subsídio ou bolsa. \\ No sponsorship or scholarship granted.
}

Recebido/Received - Junho/June 2012; Aceite/Accepted - Setembro/September 2012

Dr. Ermelindo Tavares

Serviço de Dermatologia e Venereologia

Hospital Distrital de Santarém, EPE

Avenida Bernardo Santareno

2005-177 Santarém, Portugal

Tel.: + 351963115200

Email: tavares.ermelindo@gmail.com

\section{INTRODUÇÃO}

Neisseria gonorrhoeae (gonococo) é o agente etiológico da gonorreia. Trata-se de um diplococo móvel, aeróbio, reniforme, Gram-negativo, intracelular, que infeta estritamente $o$ homem, sendo a sua transmissão sexual ou vertical. Mundialmente são diagnosticados cerca de 62 milhões de novos casos de infeção por ano. Em Portugal, a incidência foi estimada em 0.27 a 0.7 casos/100.000 habitantes ${ }^{1-5}$.

A gonorreia compreende um período de incubação de dois a cinco dias. A doença genital constitui a manifestação mais comum, sendo frequente a uretrite anterior (disúria, ardor, corrimento purulento e eritema uretral) no homem e a cervicite aguda (corrimento purulento, disúria, edema e eritema do colo uterino) na mulher; contudo, a infeção pode ser assintomática (até $10 \%$, nas uretrites; até $50 \%$, nas cervicites). O envolvimento extragenital, quando presente, traduz-se por faringite, proctite e oftalmia gonocócicas. Pode ocorrer disseminação sanguínea (gonococcemia) que, em alguns casos, resulta na síndrome dermatose-artrite ou, raramente, em meningite ou endocardite. Nas grávidas infetadas a oftalmia neonatorum é uma possível complicação no recém-nascido ${ }^{4}$.

O diagnóstico é feito recorrendo ao exame direto com coloração de Gram, à cultura em meio enriquecido e seletivo (i.e. Thayer-Martin, incubação durante $48 \mathrm{~h}$ a $37^{\circ} \mathrm{C}$ e $5-10 \%$ de $\mathrm{CO}_{2^{\prime}}$ ) e/ou a métodos moleculares relativamente simples como a hibridização ou mais complexos incluindo amplificação genética [i.e. PCR, tissue microarray (TMA)]. Atualmente, o tratamento da gonorreia é realizado de acordo com as guidelines de 2010 do Centers for Disease Control and Prevention (CDC) ou de 2009 da International Union against Sexually Transmitted Infections/World Health Organization (IUSTI/WHO) ${ }^{4,6,7}$.

O presente trabalho tem por objetivo efetuar uma revisão da bibliografia sobre resistência aos antibióticos em Neisseria gonorrhoeae, com especial enfoque nos mecanismos de resistência, dados epidemiológicos (nacionais e internacionais) e opções terapêuticas atuais e futuras.

\section{RESISTÊNCIA AOS ANTIBIÓTICOS - CARACTERIZAÇÃO GERAL}

\subsection{Aspetos gerais}

A resistência aos antibióticos em Neisseria gonorrhoeae resulta da utilização indiscriminada dos antibióticos que complica o controlo e favorece a disseminação da resistência aos mesmos. A variabilidade 
fenotípica e genética, aliadas à capacidade para incorporar novo material genético no genoma (por conjugação ou transformação) são caraterísticas intrínsecas que agravam os fenómenos de aquisição e difusão de mecanismos de resistência aos antibióticos em Neisseria gonorrhoeae ${ }^{5}$.

O aumento do número de estirpes resistentes tem várias consequências, nomeadamente, o aumento dos custos de tratamento, a realização de exames laboratoriais diferenciados e dispendiosos, a necessidade de proceder a investigação epidemiológica e a maior incidência de complicações clínicas. Como resposta a este flagelo mundial, vários países desenvolveram programas de vigilância (Tabela 1$)^{8}$.

\subsection{Mecanismos e métodos de deteção de resistência}

Vários fatores não microbianos favorecem o desenvolvimento de resistência aos antibióticos, nomeadamente escolha errada do tipo e dosagem do antibiótico, facilidade na aquisição de medicamentos (geralmente inadequados) sem prescrição médica, utilização de fármacos de qualidade duvidosa, não-adesão ao tratamento por parte do doente e/ou dos seus contactantes, ou estadias em países nos quais a resistência a determinados fármacos é endémica ${ }^{8}$.

Os mecanismos microbianos de resistência aos antibióticos são a mutação genética do próprio cromossoma, cujo aparecimento e disseminação são normalmente considerados mais lentos e/ou a aquisição genética parcial ou total de elementos extra cromossómicos (plasmídeos), mais frequente e de disseminação mais rápida. Tais fenómenos induzem resistência ao dificultar o acesso do antibiótico à proteína-alvo (por diminuição da permeabilidade celular, aumento do efluxo transmembranar, diminuição da captação microbiana e destruição e/ou neutralização do antibiótico antes de atingir o alvo) ou por causarem uma diminuição ou mesmo perda de afinidade com a proteína-alvo (por alteração da mesma) ${ }^{5,9}$.

As resistências podem ser detetadas por cultura em meios com diferentes concentrações de antibióticos e/ ou por métodos não-culturais (métodos moleculares com amplificação genética), que possibilitam a identificação do gene mutado e/ou do material genético incorporado.

\subsection{Epidemiologia}

Em termos epidemiológicos, a resistência aos antibióticos em Neisseria gonorrhoeae está dividida em três períodos: pré-quinolona, quinolona e pós-quinolona ${ }^{5}$.

\subsubsection{Era pré-quinolona}

\section{Sulfonamidas}

Entre finais do século XIX e as três primeiras décadas do século XX, o protargol (composto à base de prata)

Tabela 1 - Programas de vigilância da resistência aos antibióticos em Neisseria gonorrhoeae

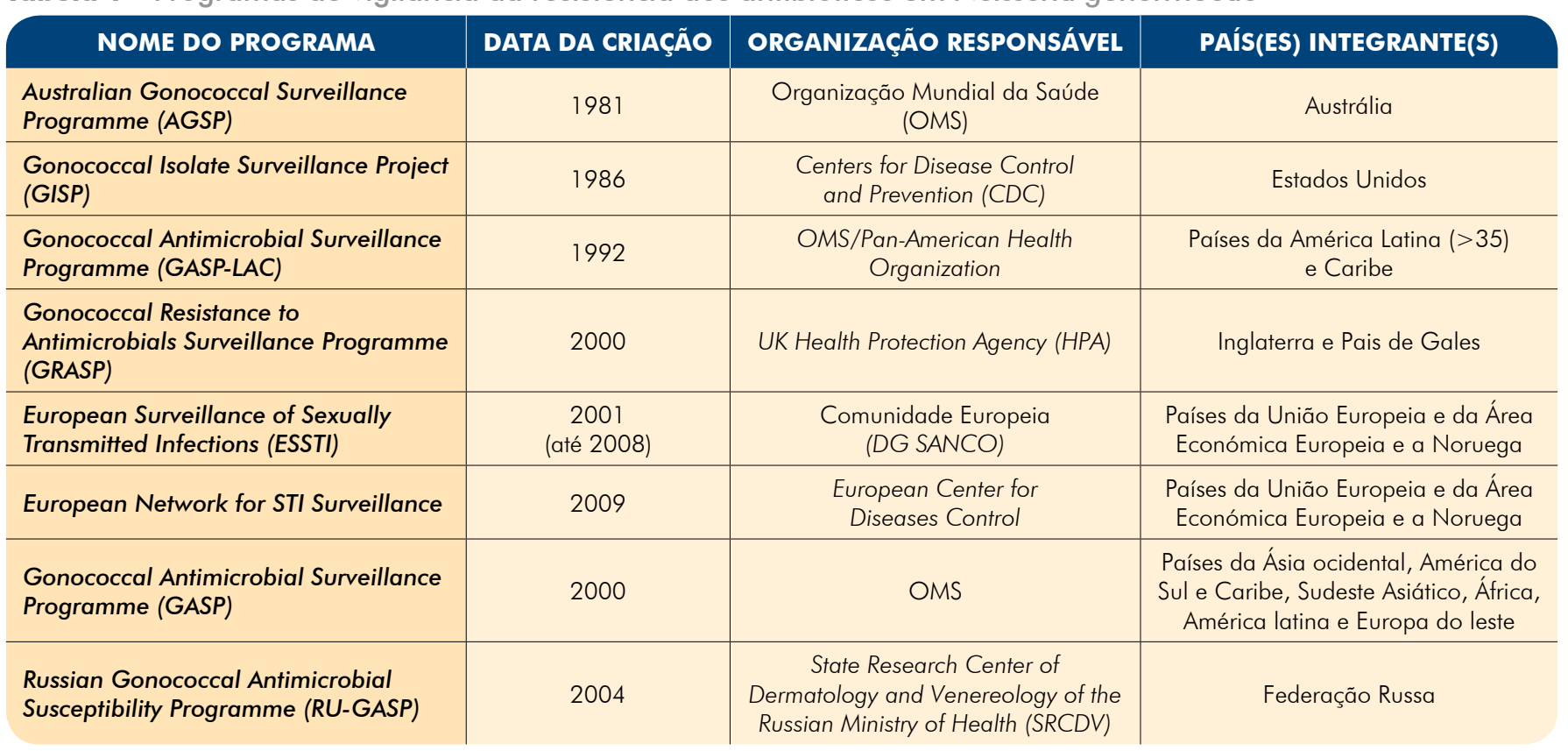

DG SANCO - Directorate General for Health and Consumer Affairs 
era o único agente disponível para o tratamento da gonorreia. A introdução da antibioterapia convencional ocorreu em 1936, com a utilização das sulfonamidas ${ }^{8}$. Sendo análogos do ácido para-aminobenzóico [para-aminobenzoic acid (PABA)], as sulfonamidas atuam por inibição competitiva da dihidropteroato sintetase (DHPS), enzima fundamental para a síntese do ácido fólico bacteriano ${ }^{10}$. Os primeiros relatos de resistência surgiram dois anos depois, tendo atingido proporções mundiais em 1944/455,9. A hiperprodução do PABA e/ ou mutação no gene que codifica a DHPS foram os principais mecanismos de resistência ${ }^{8}$. Atualmente, esta classe de antibióticos está contraindicada na maior parte dos países; contudo, a sua utilização ainda se mantém em algumas regiões subdesenvolvidas ${ }^{8,9}$.

\section{Penicilinas}

As penicilinas foram introduzidas em 1943, tendo permanecido como tratamento de primeira linha durante 40 anos $^{8}$. Através de canais e bombas proteicas codificadas por diferentes genes (pon, por, pen, $m t r$ ), as penicilinas interferem na síntese da parede celular prejudicando a divisão celular ${ }^{11}$. Durante os primeiros 10-15 anos, o aumento progressivo da concentração inibitória mínima (CIM) e o consequente aumento das doses terapêuticas, constituiu o prenúncio da emergência de estirpes resistentes. Em 1958 surgiram os primeiros relatos de resistência por mutação genética e, em 1976 foram descritas as primeiras estirpes cuja resistência foi mediada por elementos genéticos móveis (plasmídeos) $)^{5,8,9}$.

Até ao momento foram relatadas mutações genéticas nos seguintes componentes bacterianos: penA, penB, penC, ponA, $m t r R$, pem e promotor do $m t r$. No entanto, a incorporação de material genético (plasmídeos) tem sido considerada o mecanismo de resistência mais importante, ao conferir aos gonococos a capacidade de produzir $\beta$-lactamases e de, consequentemente, destruir o antibiótico. Estas estirpes foram designadas por "Neisseria gonorrhoeae produtoras de penicilinases" [Penicillinase Producing Neisseria gonorrhoeae (PPNG)] e classificadas em três grupos: África, Ásia e Rio/Toronto 8,12 .

O aparecimento e a rápida disseminação mundial das PPNG ditaram, a partir de 1989, o abandono das penicilinas como fármacos de primeira linha e a sua contra-indicação na maior parte dos países ${ }^{8,9}$.

\section{Outros antibióticos}

Durante a era pré-quinolona, os aminoglicosídeos (canamicina, gentamicina), o cloranfenicol e o seu análogo tianfenicol, os macrólidos (eritromicina e, mais tarde, a azitromicina), a espectinomicina e as tetraciclinas constituíram importantes armas terapêuticas na infeção gonocócica (Tabela 2). A resistência por mutação genética ocorreu para todas estas moléculas e, por incorporação de material extra-cromossomático (plasmídeo), apenas para as tetraciclinas. Estas últimas ficaram conhecidas por "Neisseria gonorrhoeae resistentes à tetraciclina" [Tetracycline Resistant Neisseria gonorrhoeae (TRNG) $]^{5,8,9,13,14}$.

\subsubsection{Era quinolona}

As fluoroquinolonas foram introduzidas em 1989 no tratamento da infeção gonocócica. Em 1993 três tipos (ciprofloxacina, ofloxacina, levofloxacina) foram aprovados pelo $C D C$ como fármacos de primeira linha. As quinolonas atuam por inibição da DNA girase (codificada pelo gene gyrA) e da DNA topoisomerase IV (codificada pelo gene parC), enzimas essenciais para o processo de transcrição e replicação do DNA bacteriano 9,15

Inicialmente, a CIM da ciprofloxacina era de $0.06 \mathrm{mg} / \mathrm{L}$, valor que foi aumentando gradualmente para $1 \mathrm{mg} / \mathrm{L}$ (resistência intermediária) e, por fim, $16 \mathrm{mg} / \mathrm{L}$ (estirpes resistentes) 5 $^{5}$ Estas últimas foram designadas por "Neisseria gonorrhoeae resistentes à quinolona" [Quinolone Resistant Neisseria gonorrhoeae (QRNG)]. Nos Estados Unidos, o primeiro relato de resistência à ciprofloxacina data de 1991, e foi efetuado no Havai. Casos anteriores foram descritos na Ásia, a partir da década de 1980, particularmente no Japão onde, até 1995, mais de $40 \%$ das estirpes isoladas eram resistentes ${ }^{9,16}$. A mutação do gyrA e parC, com diminuição e/ou perda da afinidade enzimática, foram os principais mecanismos de resistência ${ }^{8}$.

Em 2000 as fluoroquinolonas deixaram de ser prescritas para o tratamento das infeções por Neisseria gonorrhoeae em vários países. Em 2004 e 2005 deixaram de ser recomendadas, respetivamente, pelo CDC e pela ESSTI. Contudo, ainda são passíveis de utilização nos países com programas de vigilância onde o número de estirpes sensíveis é superior a 95\%, 87,18 .

\subsubsection{Era pós-quinolona}

Com o desuso das fluoroquinolonas, as cefalosporinas da terceira geração tornaram-se os principais fármacos no tratamento da gonorreia. O início da sua utilização remonta, no entanto, aos primórdios da década de 1980 [ceftriaxona (1980) e cefixima (1983)]. Em 2006 foram recomendadas pelo CDC como antimicrobianos de primeira linha (associadas à azitromicina), 
Tabela 2 - Caraterização dos antimicrobianos utilizados na era pré-quinolona

\begin{tabular}{|c|c|c|c|c|c|c|}
\hline \multirow{2}{*}{ ANTIBIÓtICO } & \multirow{2}{*}{ INTRODUÇÃO } & \multirow{2}{*}{$\begin{array}{l}\text { MECANISMO } \\
\text { DE AÇÃOO }\end{array}$} & \multirow{2}{*}{$\begin{array}{l}\text { RESISTÊNCIA } \\
\text { (primeiros } \\
\text { relatos) }\end{array}$} & \multicolumn{2}{|c|}{ MECANISMOS DE RESISTÊNCIA } & \multirow{2}{*}{$\begin{array}{l}\text { INDICAÇÃO } \\
\text { ATUAL }\end{array}$} \\
\hline & & & & Mutação genética & Plasmídeo & \\
\hline Aminoglicosídeos ${ }^{8,9}$ & 1949 & $\begin{array}{l}\text { Inibição de } \\
\text { síntese proteica } \\
\text { - Interfere com } \\
\text { a subunidade } \\
\text { ribossomal 30S }\end{array}$ & 1958 & $\begin{array}{l}\text { Gene: Kan } \\
\text { Resultado: Inativação } \\
\text { do antibiótico }\end{array}$ & $\begin{array}{l}\text { Não } \\
\text { relatado }\end{array}$ & $\begin{array}{l}\text { Não recomendados } \\
\text { Canamicina e } \\
\text { Gentamicina são } \\
\text { utilizadas em } \\
\text { alguns países } \\
\text { subdesenvolvidos }\end{array}$ \\
\hline Macrólidos $8,9,14$ & $\begin{array}{l}\text { Eritromicina } \\
1952 \\
\text { Azitromicina } \\
1983\end{array}$ & $\begin{array}{l}\text { Inibição de } \\
\text { síntese proteica } \\
\text { - Interfere com } \\
\text { a subunidade } \\
\text { ribossomal 50S }\end{array}$ & $\begin{array}{l}\text { Eritromicina - } \\
\text { S.D. } \\
\text { Azitromicina - } \\
1999\end{array}$ & $\begin{array}{l}\text { Genes: 23sRNArrl, } \\
\text { mtrC, mtrR, ermB, ermC, } \\
\text { ermF, mef, gyrA, parC, } \\
\text { promotor do mtrR e mtrC } \\
\text { Resultado: Aumento do } \\
\text { efluxo transmembranar, } \\
\text { inativação (metilação), } \\
\text { diminuição da captação, } \\
\text { diminuição/perda de } \\
\text { afinidade }\end{array}$ & $\begin{array}{l}\text { Não } \\
\text { relatado }\end{array}$ & $\begin{array}{l}\text { Azitromicina - } \\
\text { Coadjuvante das } \\
\text { cefalosporinas } \\
\text { Eritromicina - Não } \\
\text { recomendada }\end{array}$ \\
\hline Espectinomicina $a^{8,9,13}$ & 1961 & $\begin{array}{l}\text { Inibição de } \\
\text { síntese proteica } \\
\text { - Interfere com } \\
\text { a subunidade } \\
\text { ribossomal 30S }\end{array}$ & 1987 & $\begin{array}{l}\text { Gene: } s p c \\
\text { Resultado: Diminuição } \\
\text { da afinidade }\end{array}$ & $\begin{array}{l}\text { Não } \\
\text { relatado }\end{array}$ & $\begin{array}{l}\text { Alternativa às } \\
\text { cefalosporinas }\end{array}$ \\
\hline Tetraciclinas $s^{5,8,9}$ & 1962 & $\begin{array}{l}\text { Inibição de } \\
\text { síntese proteica } \\
\text { - Interfere com } \\
\text { a subunidade } \\
\text { ribossomal 30S }\end{array}$ & $\begin{array}{c}\text { Mutação } \\
\text { genética-- } \\
1970 \\
\text { Plasmídeo } \\
\text { (TRNG) - } 1985\end{array}$ & $\begin{array}{l}\text { Genes: rpsJ, penB, mtrR, } \\
\text { promotor do mtrR, tem } \\
\text { Resultados: } \\
\text { Diminuição/perda de } \\
\text { afinidade, aumento } \\
\text { do efluxo celular, } \\
\text { diminuição da captação }\end{array}$ & tet-M & Não recomendadas \\
\hline
\end{tabular}

S.D. - Sem dados; TRNG - Tetracycline Resistant Neisseria gonorrhoeae

situação que ainda se mantém. Tal como as penicilinas, as cefalosporinas inibem a síntese da parede celular por inibição das enzimas de transpeptidação. Atuam por interferência com as proteínas de ligação às penicilinas e com canais e bombas proteicas codificadas, respetivamente, pelos genes penA, penB e $m t r^{5,8,9,19}$.

Atualmente são ainda poucos os casos relatados de resistência ${ }^{20-22}$, sendo o aumento da CIM (ceftriaxone $\geq 0.125 \mathrm{mg} / \mathrm{L}$ e cefixima $\geq 0.25 \mathrm{mg} / \mathrm{L}$ ), traduzido pela diminuição da sensibilidade, o evento mais frequente em várias partes do mundo, após os primeiros relatos originários do Japão $0^{5,8,9}$. Tal facto deveu-se principalmente à mutação nos genes penA, pen $B$, ponA, mtrR e promotor do mtrR, resultando numa diminuição da captação e num aumento do efluxo transmembranar dos antibióticos pelas bactérias ${ }^{8,9}$.

\section{RESISTÊNCIA AOS ANTIBIÓTICOS - DADOS EPIDEMIOLÓGICOS}

\subsection{Dados nacionais}

O primeiro caso de PPNG em Portugal foi relatado por Torgal Garcia et al. ${ }^{23}$, em 1982, tendo sido observado na valência de venereologia, mais conhecida como "Consulta das Francesinhas", do atual Centro de Saúde da Lapa. Tratava-se de uma mulher de 29 anos, que praticava prostituição, que alegou não ter tido contactos com indivíduos de nacionalidade estrangeira e com quatro episódios de gonorreia e três de sífilis recente nos seus antecedentes clínicos, observada em consulta de rotina. Apesar de não referir sintomatologia, ao exame ginecológico constatou-se exsudado escasso e não purulento no colo. As gonoculturas foram positivas, 


\section{GEIDST}

tendo sido observado crescimento de uma estirpe de Neisseria gonorrhoeae produtora de penicilinases mas sensível à espectinomicina, a qual foi usada no tratamento (duas injeções intramusculares, $2 \mathrm{~g}+2 \mathrm{~g}$ ), tendo promovido a cura da infeção.

A partir de 1982 registou-se um aumento progressivo dos casos de resistência às penicilinas, facto constatado em dois grandes estudos realizados por Vaz Pato et al. ${ }^{24}$ e Vieira et al. ${ }^{25}$. No primeiro, $1.1 \%$ em 1983, $8.2 \%$ em 1984, $19.2 \%$ em 1985 e $27 \%$ em 1987 eram estirpes PPNG; a determinação do tipo de plasmídeo demonstrou que $71 \%$ eram do tipo África e $26 \%$ do tipo Ásia. No estudo de Vieira et al. ${ }^{25}$, foram detetadas $11 \%$ de estirpes PPNG em 1987, $21 \%$ em 1988, 36\% em 1989 e $37 \%$ em 1990.

O primeiro caso de TRNG (CIM $\geq 16 \mathrm{mg} / \mathrm{L})$ foi descrito em 1990, em Lisboa, mas na sequência do estudo realizado por Ferreira et al. ${ }^{26}$, verificou-se um aumento progressivo e assinalável do número TRNG entre 1991 e 1995, de $4 \%$ em 1991, $5.3 \%$ em 1992, $10.8 \%$ em 1994, até $52.2 \%$ em 1995 . Acresce que $78.3 \%$ das TRNG eram igualmente produtoras de penicilinases.

Mais recentemente, o estudo realizado por Florindo et al. ${ }^{27}$, com estirpes isoladas entre 2004 e 2009, mostrou que $37.4 \%$ eram resistentes à ciprofloxacina $(\mathrm{CIM} \geq 1 \mathrm{mg} / \mathrm{L})$ e $79.1 \%$ às penicilinas $(\mathrm{CIM} \geq 2 \mathrm{mg} / \mathrm{L})$, sendo que destas últimas $15.5 \%$ eram PPNG. Não foram observadas resistências à espectinomicina ou à ceftriaxona; contudo, quatro revelaram diminuição da sensibilidade $(0.125 \geq M I C \leq 0,25 \mathrm{mg} / \mathrm{L})$ a este último antibiótico.

Em relação à azitromicina, de acordo com os dados do ESSTI (European Surveillance of Sexually Transmitted
Infections), em Portugal as taxas de resistência a este macrólido em 2006, 2007 e 2008 foram de 6.9\%, $8.3 \%$ e $0 \%$, respetivamente ${ }^{28}$.

\subsection{Dados internacionais}

Nos Estados Unidos (Tabela 3) 29-31, e de acordo com - Gonococcal Isolate Surveillance Project (GISP) para os anos 2003, 2006 e 2007, as penicilinas, as tetraciclinas e as quinolonas foram os antibióticos com maior número de resistências. Contudo, entre 2003 e 2007 não houve casos de resistência às cefalosporinas, espectinomicina e azitromicina. Embora ligeiro, entre 2000 e 2010 constatou-se um aumento dos casos de diminuição da sensibilidade à cefixima $(C I M \geq 0.125 \mathrm{mg} / \mathrm{L})$ e ceftriaxona $(\mathrm{CIM} \geq 0.25 \mathrm{mg} / \mathrm{L})$, na sua maioria em homens que fazem sexo com homens.

Em Inglaterra (Tabela 4) ${ }^{32}$, relatórios recentes do Gonococcal Resistance to Antimicrobials Surveillance Programme (GRASP) revelaram casos de resistência à azitromicina mas não foram isoladas estirpes de Neisseria gonorrhoeae resistentes às cefalosporinas de terceira geração; no entanto, foram detetadas estirpes com redução da sensibilidade à cefixima e ceftriaxona, predominantemente nos homens que fazem sexo com homens $(33.4 \%$, em comparação aos $5.7 \%$ nos homens que não fazem sexo com homens), tal como acima descrito para os Estados Unidos.

Nos países que integraram o European Surveillance of Sexually Transmitted Infections (ESSTI) (Tabela 5) 33,28, a ciprofloxacina foi o antimicrobiano com maior número de estirpes resistentes nos anos 2008 e 2009. Não foram reportados casos de resistência às cefalosporinas de terceira geração ou à espectinomicina.

Tabela 3 - Resistência aos antibióticos em Neisseria gonorrhoeae. Dados epidemiológicos gerais de acordo com o GISP (Estados Unidos)

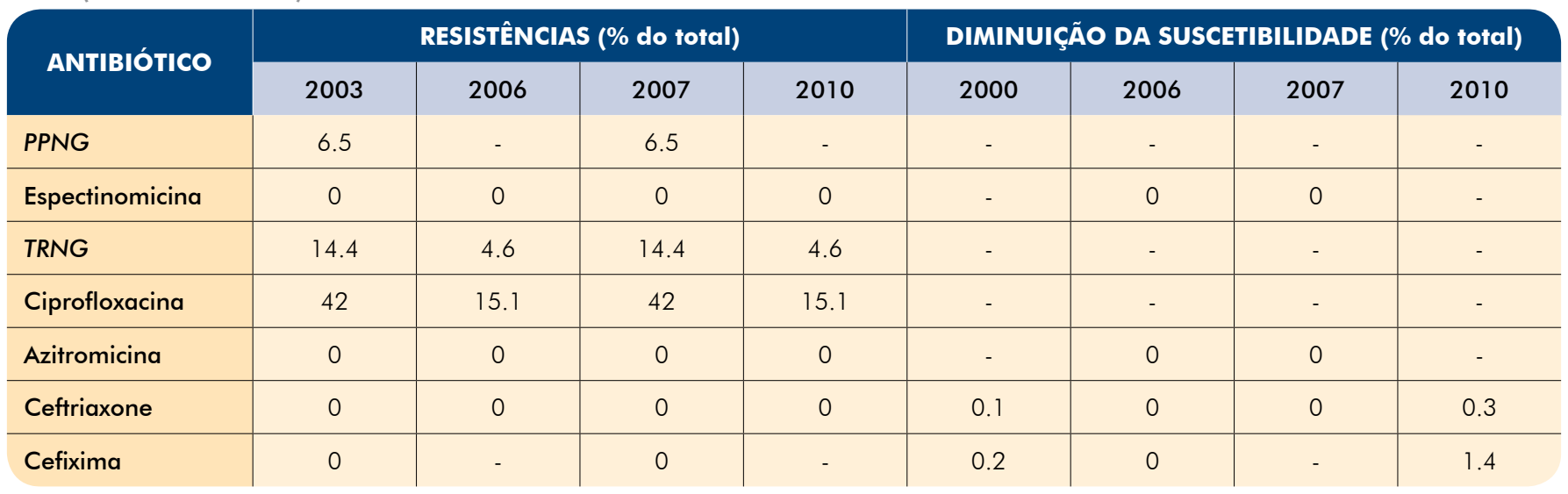

TRNG - Tetracycline Resistant Neisseria gonorrhoeae; PPNG - Penicillinase Producing Neisseria gonorrhoeae 
Tabela 4 - Resistência aos antibióticos em Neisseria gonorrhoeae. Dados epidemiológicos gerais de acordo com o GRASP (Inglaterra e País de Gales)

\begin{tabular}{l|c|c|c|c|}
\hline \multirow{2}{*}{ ANtIBIÓtIco } & \multicolumn{2}{|c|}{ RESISTÊNCIAS (\% do total) } & \multicolumn{2}{c|}{ DIMINUIÇÃO DA SUSCETIBILIDADE (\% do fotal) } \\
\cline { 2 - 5 } & $\mathbf{2 0 0 9}$ & $\mathbf{2 0 1 0}$ & $\mathbf{2 0 0 9}$ & $\mathbf{2 0 1 0}$ \\
\hline PPNG & 5.2 & 5.5 & - & - \\
\hline Ciprofloxacina & 35.3 & 35.7 & - & - \\
\hline Azitromicina & 1.2 & 0.5 & 0.3 & 0 \\
\hline Ceftriaxone & 0 & 0 & 6.1 & 9.6 \\
\hline
\end{tabular}

PPNG - Penicillinase Producing Neisseria gonorrhoeae

Tabela 5 - Resistência aos antibióticos em Neisseria gonorrhoeae. Dados epidemiológicos gerais de acordo com o ESSTI (Países da União Europeia e Comunidade Económica Europeia)

\begin{tabular}{l|c|c|c|c}
\hline \multirow{2}{*}{ ANTIBIÓtICO } & \multicolumn{2}{|c|}{ RESISTÊNCIAS (\% do total isolado) } & \multicolumn{2}{c}{ DIMINUIÇÃO DA SUSCETIBILIDADE (\% do total isolado) } \\
\hline PPNG & 2008 & 2009 & 2008 & 2009 \\
\hline Espectinomicina & 12 & 12 & - & - \\
\hline TRNG & - & 0 & - & - \\
\hline Ciprofloxacina & 16 & - & - & - \\
\hline Azitromicina & 51 & 63 & - & - \\
\hline Ceftriaxone & 1.9 & 13 & 0 & 0 \\
\hline \multicolumn{2}{c|}{ Cefixima } \\
TRNG - Tetracycline Resistant Neisseria gonorrhoeae; PPNG - Penicillinase Producing Neisseria gonorhoeae
\end{tabular}

O maior número de microrganismos com diminuição da suscetibilidade à cefixima foi observado na Áustria (21.2\%), na ltália (18.6\%) e na Dinamarca (15.1\%). Em relação à azitromicina, a Dinamarca (46\%), a Áustria (29\%), a Itália (29\%) e a França (19\%), apresentaram o maior número de estirpes resistentes.

Na Rússia, de acordo com o Russian Gonococcal Antimicrobial Susceptibility Programme (RU-GASP) para os anos de 2007 e 2008, o número de estirpes resistentes foi, respetivamente, de $49.6 \%$ e $49.1 \%$ para a ciprofloxacina, $0.9 \%$ e $7.2 \%$ para a espectinomicina e $2.3 \%$ e $0.4 \%$ para a azitromicina. Não foram reportados casos de resistência à ceftriaxona ou sequer de diminuição da respetiva $\mathrm{CIM}^{34}$.

Na Austrália ${ }^{35}$, segundo a Australian Government Publishing Service (AGSP) do ano 2010, apenas 3\% das estirpes eram sensíveis à ciprofloxacina mas todas se revelaram suscetíveis à espectinomicina e à ceftriaxona.
Dos 11 países da América Latina com dados disponíveis no relatório do Gonococcal Antimicrobial Susceptibility Surveillance Program in Latin america and Caribbean (GASP-LAC) para o ano de 2009, nenhum reportou casos de resistência à espectinomicina ou à ceftriaxona ${ }^{36}$.

\section{TRATAMENTO DA GONORREIA NA ERA DA RESISTÊNCIA AOS ANTIBIÓTICOS}

\subsection{Terapêutica atual}

Atualmente preconiza-se o tratamento da gonorreia de acordo com as recomendações de duas guidelines internacionais: CDC $2010^{6}$ e IUSTI/WHO 20097 (Tabela 6). Na primeira é recomendada a ceftriaxona ( $250 \mathrm{mg}, \mathrm{IM}$ ) ou a cefixima (400mg, per os), associadas à azitromicina ( $1 \mathrm{~g}$, per os), em dose única, como terapêutica de primeira linha para a infeção gonocócica 


\section{GEIDST}

Tabela 6 - Guidelines norte americana (Estados Unidos - CDC 2010) e europeia (IUSTI/WHO 2009) para o tratamento da infeção gonocócica aguda não complicada (cervicite, uretrite e proctite)

\begin{tabular}{|c|c|c|}
\hline \multirow{2}{*}{ GUIDELINE } & \multicolumn{2}{|l|}{ TRATAMENTO RECOMENDADO } \\
\hline & Primeira linha & Alternativa \\
\hline $\begin{array}{c}\text { CDC } 2010 \\
\text { (Estados Unidos) }\end{array}$ & $\begin{array}{l}\text { Ceftriaxona } 250 \mathrm{mg} \text {, IM (dose única) ou Cefixima } 400 \mathrm{mg} \text {, po (dose única) + } \\
\text { Azitromicina lg po (dose única) ou Doxicilina } 100 \mathrm{mg} \text { po (7 dias) }\end{array}$ & Azitromicina 2g, po (dose única) \\
\hline $\begin{array}{l}\text { IUSTI/WHO } 2009 \\
\text { (Europeia) }\end{array}$ & $\begin{array}{c}\text { Ceftriaxona } 250 \mathrm{mg} \text {, IM (dose única) ou Cefixima } 400 \mathrm{mg} \text {, po (dose única) ou } \\
\text { Espectinomicina } 2 \mathrm{~g} \text {, IM (dose única) }\end{array}$ & $\begin{array}{l}\text { Outras cefalosporinas da terceira geração } \\
\text { (dose única) }\end{array}$ \\
\hline
\end{tabular}

aguda não complicada (uretrite, cervicite e proctite). A IUSTI/WHO aconselha igualmente uma dose única de ceftriaxona $(250 \mathrm{mg}, I M)$ ou cefixima ( $400 \mathrm{mg}$, per os), embora sem associação à azitromicina, e inclui a espectinomicina $(2 g, I M)$ como alternativa terapêutica.

\subsection{Terapêuticas do futuro}

Com o intuito de impedir que a infeção por Neisseria gonorrhoeae venha a carecer de alternativas terapêuticas foram apontadas, até ao momento, várias soluções, com destaque para a criação e implementação de novas estratégias de utilização dos antibióticos e para o desenvolvimento de novos antimicrobianos.

\subsubsection{Alternativas baseadas nas terapêuticas atuais}

\section{Aumento da dose das cefalosporinas}

O aumento da dose do ceftriaxona tem sido considerada uma alternativa terapêutica eficaz nos casos de diminuição da sensibilidade, tendo sido proposta pela British Association of Sexual Health and HIV (BASHH) ${ }^{37}$ e por Chisholm et al. ${ }^{38}$, que recomendaram uma administração intramuscular (IM) de $500 \mathrm{mg}$ e uma IM ou endovenosa (EV) de $1 \mathrm{~g}$, respetivamente. Contudo, outros autores consideram que tal medida apenas produzirá efeitos por um curto espaço de tempo", tal como no passado aconteceu com as penicilinas. No caso da cefixima, uma das principais limitações do aumento da dose terapêutica para valores acima dos $400 \mathrm{mg}$ seriam os eventuais efeitos adversos a nível gastrointestinal. No entanto, um estudo recente ${ }^{39}$ propõe a administração da cefixima em $2 \times 400 \mathrm{mg}$, com intervalo de 6 horas entre as tomas.

\section{Associação de antibióticos}

Foram sugeridas várias associações de antibióticos para o tratamento da infeção por estirpes resistentes de Neisseria gonorrhoeae. Chisholm et al. ${ }^{38}$ propuseram os seguintes esquemas: ceftriaxona $\lg \mathrm{IM}$, dose única, seguida de cefixima oral $400 \mathrm{mg}$ por dia durante dois dias ou de azitromicina oral $2 \mathrm{~g}$, dose única. Lewis et al. ${ }^{8}$ sugeriram a combinação de espectinomicina $2 \mathrm{~g} \mathrm{IM}$ e azitromicina oral $2 \mathrm{~g}$, ambas em dose única, como alternativa à ceftriaxone $250 \mathrm{mg}$ nos casos de resistência à cefixima. Por fim, a associação de gentamicina e azitromicina, de acordo com Unemo et al. ${ }^{9}$, é uma opção terapêutica promissora.

\subsubsection{Alternativas baseadas em novas terapêu-} ticas

O ertapenem, uma molécula relativamente recente da família dos carbapenemas, poderia constituir uma alternativa terapêutica nos casos de resistência à ceftriaxona; contudo, a ineficácia observada quando tal resistência é mediada pelo gene PenA retiram consistência à hipótese de utilizar o ertapenem como uma alternativa universal ${ }^{39}$.

Estirpes de Neisseria gonorrhoeae multirresistentes revelaram ser sensíveis a novos agentes farmacológicos, na sua maioria experimentais, pelo que a utilização destas substâncias, enquanto alternativas terapêuticas, poderá vir a ser equacionada no futuro (Tabela 7). Destacamos:

- Os inibidores da UDP-3-O-(acil)-N-acetilglucosamina diacetilase $(L p x C)$ que demonstraram atividade in vitro contra várias bactérias Gram-negativas multirresistentes, incluindo os gono$\operatorname{cocos}^{40}$;

- O peptídeo antimicrobiano LL-37 (da família das catelicidinas humanas), já que alguns estu$\operatorname{dos}^{41}$ demonstraram a sua atividade bactericida e imunomoduladora (prevenção da endotoxemia e apoptose neutrofílica);

- Os aminocoumarinos, nomeadamente as novobiocina, clorobiocina e coumermicina, antibacterianos naturais (obtidos a partir de espécies de Streptomyces) que inibem a DNA girase de Neisseria gonorrhoeae ${ }^{42}$;

- O eugenol, um antissético natural extraído da planta Ocimum sanctum, cuja atividade antimicrobiana, particularmente em estirpes 
Tabela 7 - Infeção por Neisseria gonorrhoeae multirresistente - Possíveis alternativas terapêuticas no futuro

\begin{tabular}{|c|c|c|c|}
\hline $\begin{array}{l}\text { DESIGNAÇÃO } \\
\text { DA SUBSTÂNCIA }\end{array}$ & CLASSE & $\begin{array}{l}\text { MECANISMO } \\
\text { DE AÇÃOO }\end{array}$ & $\begin{array}{c}\text { DISPONIBILIDADE } \\
\text { ATUAL }\end{array}$ \\
\hline CHIR-090 & $\begin{array}{l}\text { Inibidor da UDP-3-O-(acil)-N- } \\
\text { acetilglucosamina diacetilase }(L p \times C)\end{array}$ & $\begin{array}{l}\text { Inibição da biossíntese do lípido A, componente } \\
\text { hidrofílico do lipoligossacarídeo (LPS) }\end{array}$ & Experimental \\
\hline LL-37 & Peptídeo catelicidina antimicrobiano & $\begin{array}{l}\text { Antibacteriano direto (neutralização do LPS) } \\
\text { Imunomodulador (Inibição da apoptose } \\
\text { neutrofílica) }\end{array}$ & Experimental \\
\hline H12c (Eugenol) & - & Coagulação proteica & Experimental \\
\hline CEM-112 (Solitromicina) & Macrólidos & $\begin{array}{l}\text { Interferência com subunidade ribossomal 50S, } \\
\text { inibindo a síntese proteica }\end{array}$ & Estudo de fase II \\
\hline
\end{tabular}

multirresistentes de Neisseria gonorrhoeae, foi evidenciada em ratos $^{43}$;

- A solitromicina, um novo macrólido desenvolvido para as pneumonias adquiridas na comunidade, nomeadamente as causadas por bactérias multirresistentes, que revelou igualmente ter elevada atividade, in vitro, em estirpes de Neisseria gonorrhoeae multirresistentes ${ }^{44}$;

- A Terminalia macroptera, uma planta africana com várias aplicações medicinais, cuja atividade antimicrobiana em várias estirpes de Neisseria gonorrhoeae, incluindo PPNG e TRNG, foi demonstrada por Silva et al. ${ }^{45}$.

O desenvolvimento de uma vacina para Neisseria gonorrhoeae é dificultado pela elevada variabilidade genómica e antigénica da bactéria, pelo que não se prevê que uma vacina capaz de induzir uma proteção eficaz e duradoura esteja a breve trecho disponível.

\section{CONCLUSÃO}

A infeção por estirpes de Neisseria gonorrhoeae resistentes aos antibióticos acarreta elevados custos para os sistemas de saúde e pode ter consequências profundas para o doente. Como tal, a resistência aos antimicrobianos em Neisseria gonorrhoeae foi, e continua a ser, um importante problema em termos de saúde pública, sendo imperativo que novas e melhores estratégias de controlo sejam elaboradas e implementadas.

No caso de Portugal, consideram-se como fatores cruciais: primeiro, a vigilância epidemiológica eficaz, sobretudo das populações em maior risco; segundo, o diagnóstico laboratorial das infeções por Neisseria gonorrhoeae; terceiro, a pesquisa da sensibilidade aos antibióticos; quarto, o tratamento adequado dos doentes e dos seus contactantes, sempre que possível com controlo da eficácia terapêutica; quinto, a utilização correta do antibiótico (tipo e dose); sexto, a notificação dos casos de gonorreia; e, por último, a caracterização das estirpes de Neisseria gonorrhoeae isoladas em Portugal (promovida desde 2006 pelo Instituto Nacional de Saúde Dr. Ricardo Jorge de acordo com as recomendações do European Center for Diseases Control, $\mathrm{ECDC})$.

\section{REFERÊNCIAS}

1. Todar K. Pathogenic Neisseriae: Gonorrhea, Neonatal Ophthalmia and Meningococcal Meningitis. Todar's online textbook of bacteriology. Disponível em: http://www.textbookofbacteriology.net/neisseria.html. [acedido em 2012 Maio 6].

2. World Health Organization Regional Office for Europe. Gonorrhoea - incidence rate (per 100,000 population). Copenhagen: WHO Regional Office for Europe; 2010.

3. Tapsall JW. What management is there for gonorrhea in the post-quinolone era. Sex Transm Dis. 2006; 33:8-10.

4. Stary A. Sexually transmitted infections. In: Bolognia JL, Jorizzo JL, Rapini RP, et al, editores. Dermatology. Madrid: Mosby Elsevier; 2008. p. 1239-61. 
5. Patel AL, Chaudhry U, Sachdev D, Sachdeva PN, Bala M, Saluja D. An insight into the drug resistance profile and mechanism of drug resistance in Neisseria gonorrhoeae. Indian J Med Res. 2011; 134:419-31.

6. Workowski KA, Berman S; Centers for Disease Control and Prevention (CDC). Sexually transmitted diseases treatment guidelines, 2010. MMWR Recomm Rep. 2010 17; 59(RR-12):1-110.

7. Bignell C; IUSTI/WHO. 2009 European (IUSTI/ WHO) guideline on the diagnosis and treatment of gonorrhoea in adults. Int J STD AIDS. 2009; 20(7):453-7.

8. Lewis DA, Lukehart SA. Antimicrobial resistance in Neisseria gonorrhoeae and Treponema pallidum: evolution, therapeutic challenges and the need to strengthen global surveillance. Sex Transm Infect. 2011; 87 Suppl 2: ii39-43.

9. Unemo M, Shafer WM. Antibiotic resistance in Neisseria gonorrhoeae: origin, evolution, and lessons learned for the future. Ann N Y Acad Sci. 2011; 1230:E19-28.

10. Sulphonamide (medicine). Wikipédia.org [homepage na Internet]. Disponível em: http://en.wikipedia. org/wiki/Sulfonamide_medicine. [acedido em 2012 Maio 6].

11. Penicilin. Wikipédia.org [homepage na Internet]. Disponível em: http://en.wikipedia.org/wiki/Penicillin. [acedido em 2012 Maio 6].

12. Ohnishi M, Ono E, Shimuta K, Watanabe H, Okamura N. Identification of TEM-135 beta-lactamase in penicillinase-producing Neisseria gonorrhoeae strains in Japan. Antimicrob Agents Chemother. 2010; 54(7):3021-3.

13. Maness MJ, Foster GC, Sparling PF. Ribosomal resistance to streptomycin and spectinomycin in Neisseria gonorrhoeae. J Bacteriol. 1974; 120(3):1293-9.

14. Cousin S Jr., Whittington WL, Roberts MC. Acquired macrolide resistance genes in pathogenic Neisseria spp. isolated between 1940 and 1987. Antimicrob Agents Chemother. 2003; 47(12):3877-80.

15. Quinolones. Wikipédia.org [homepage na Internet]. Disponível em: http://en.wikipedia.org/wiki/ Quinolone [acedido em 2012 Maio 6].

16. Tanaka M, Kumazawa J, Matsumoto T, Kobayashi I. High prevalence of Neisseria gonorrhoeae strains with reduced susceptibility to fluoroquinolones in Japan. Genitourin Med. 1994; 70:90-3.

17. Martin IM, Hoffmann S, Ison CA; ESSTI Network. European Surveillance of Sexually Transmitted Infections (ESSTI): the first combined antimicrobial susceptibility data for Neisseria gonorrhoeae in Western Europe. J Antimicrob Chemother. 2006; 58:587-93.

18. Centers for Disease Control and Prevention. Update to CDC's sexually transmitted diseases treatment guidelines, 2006: fluoroquinolones no longer recommended for treatment of gonococcal infections. MMWR Morb Mortal Wkly Rep. 2007; 56:332-6.

19. Cephalosporin. Wikipédia.org [homepage na Internet]. Disponível em: http://en.wikipedia.org/ wiki/Cephalosporin [acedido em 2012 Maio 6].

20. Suzaki A, Hayashi K, Kosuge K, Soma M, Hayakawa S. Disseminated gonococcal infection in Japan: a case report and literature review. Intern Med. 2011 ; 50(18):2039-43.

21. Carnicer-Pont D, Smithson A, Fina-Homar E, Bastida MT; Gonococcus Antimicrobial Resistance Surveillance Working Group. First cases of Neisseria gonorrhoeae resistant to ceftriaxone in Catalonia, Spain. Enferm Infecc Microbiol Clin. 2012; 30(4):218-9.

22. Unemo M, Golparian D, Nicholas R, Ohnishi $M$, Gallay A, Sednaoui P. High-level cefixime- and ceftriaxone-resistant Neisseria gonorrhoeae in France: novel penA mosaic allele in a successful international clone causes treatment failure. Antimicrob Agents Chemother. 2012; 56(3):1273-80.

23. Torgal Garcia J, Vaz Pato MV, Ribeiro Pires I, Prates Nunes F. Isolamento da primeira estirpe de Neisseria gonorrhoeae produtora de beta-lactamase, penicilino-resistente, em Portugal. Trab Soc Port Dermatol Venereol. 1984; 42 (2):79-84.

24. Vaz Pato MV, Ribeiro Pires I, Van Klingeren B, Louro D, Rosa H. Neisseria gonorrhoeae productrices de penicillinase (NGPP) isolees a Lisbonne 19821987 . Pathol Biol. 1988; 36(5 Pt 2):639-42.

25. Vieira R, Conchon I, Ferreira J, Medeiros MJ, Lacerda MH. Gonorreia-Infecções por Estirpes Produtoras de Penicilinase. Consulta de Doenças de Transmissão Sexual. Serviço de Dermatovenereologia. Hospital de Curry Cabral. 1987-1990. Trab Soc Port Dermatol Venereol. 1991; 49 (4):223-8.

26. Ferreira E, Louro D, Gomes JP, Catry MA, Pato MV. High-level tetracycline resistant Neisseria gonorrhoeae isolated in Portugal. Pathol Biol. 1997; 45(5):371-5.

27. Florindo $C$, Pereira R, Boura $M$, Nunes $B$, Paulino A, Gomes JP, et al. Genotypes and antimicrobial-resistant phenotypes of Neisseria gonorrhoeae in Portugal (2004-2009). Sex Transm Infect. 2010; 86(6):449-53. 
28. Cole MJ, Chisholm SA, Hoffmann S, Stary A, Lowndes CM, Ison CA; European Surveillance of Sexually Transmitted Infections Network.. European surveillance of antimicrobial resistance in Neisseria gonorrhoeae. Sex Transm Infect. 2010; 86(6):427-32.

29. Wang SA, Harvey AB, Conner SM, Zaidi AA, Knapp JS, Whittington WL, et al. Antimicrobial resistance for Neisseria gonorrhoeae in the United States, 1988 to 2003: the spread of fluoroquinolone resistance. Ann Intern Med. 2007; 147(2):81-8.

30. CDC.gov [homepage na Internet]. Gonococcal Isolate Surveillance Project (GISP) Annual Report 2007. Disponível em http://www.cdc.gov/std/ gisp2007/GISPSurvSupp2007Complete.pdf. [Acedido em 2012 Maio 6].

31. Centers for Disease Control and Prevention (CDC). Cephalosporin susceptibility among Neisseria gonorrhoeae isolates-United States, 2000-2010. MMWR Morb Mortal Wkly Rep. 2011 ; 60(26):873-7.

32. HPA. Gov. uk. [homepage na Internet]. GRASP 2010 Report. Disponível em: http://www.hpa.org.uk/ webc/HPAwebFile/HPAweb_C/1316016752917. [Acedido em 2012 Maio 12].

33. Cole MJ, Unemo M, Hoffmann S, Chisholm SA, Ison CA, van de Laar MJ. The European gonococcal antimicrobial surveillance programme, 2009. Euro Surveill. 2011; 16(42). pii: 19995.

34. Kubanova A, Frigo N, Kubanov A, Sidorenko S, Lesnaya I, Polevshikova $S$, et al. The Russian gonococcal antimicrobial susceptibility programme (RU-GASP)-national resistance prevalence in 2007 and 2008, and trends during 2005-2008. Euro Surveill. 2010; 15(14). pii: 19533.

35. Australian Gonococcal Surveillance Programme. Annual Report of the Australian Gonococcal Surveillance Programme, 2009. Commun Dis Intell. 2010; 34(2):89-95.

36. GASP-LAC.net [homepage na Internet]. Report of the Workshop for the Revitalization of the Gonococcal Antimicrobial Susceptibility Surveillance Program in Latin America and the Caribbean (GASP-LAC). Disponível em: http://www.gasp-lac. net/docs/GASP-LAC_Meeting_Report_FINAL_Addendum.pdf. [Acedido em 2012 Maio 12].
37. Sacks R, Greene L. New management of gonorrhoea. Impact of antibiotic resistance. BMJ. 2011; 342: d3523.

38. Chisholm SA, Mouton JW, Lewis DA, Nichols T, Ison CA, Livermore DM. Cephalosporin MIC creep among gonococci: time for a pharmacodynamic rethink? J Antimicrob. Chemother. 2010; 65:2141-8.

39. Unemo M, Golparian D, Limnios A, Whiley D, Ohnishi $M$, Lahra $M M$, et al. In vitro activity of ertapenem vs. ceftriaxone against Neisseria gonorrhoeae isolates with highly diverse ceftriaxone MIC values and effects of ceftriaxone resistance determinants ertapenem for treatment of gonorrhea? Antimicrob Agents Chemother. $2012 ; 56(7): 3603-9$

40. Zhang J, Zhang L, Li X, XU W. UDP-3-O-(R-3-hydroxymyristoyl)-N-acetylglucosamine deacetylase (LpxC) inhibitors: a new class of antibacterial agents. Curr Med Chem. 2012; 19 (13):2038-50.

41. Bucki R, Bucki R, Leszczyńska K, Namiot A, Sokołowski W. Cathelicidin LL-37: a multitask antimicrobial peptide. Arch Immunol Ther Exp. 2010; 58(1):15-25.

42. Marcu MG, Schulte TW, Neckers L.. Novobiocin and related coumarins and depletion of heat shock protein 90-dependent signaling proteins. J Natl Cancer Inst. 2000; 92(3):242-8.

43. Shokeen P, Shokeen P, Bala M, Singh $M$, Tandon. In vitro activity of eugenol, an active component from Ocimum sanctum, against multiresistant and susceptible strains of Neisseria gonorrhoeae. Int J Antimicrob Agents. 2008; 32(2):174-9.

44. Golparian D, Fernandes P, Ohnishi M, Jensen JS, Unemo M. In vitro activity of the new fluoroketolide solithromycin (CEM-101) against a large collection of clinical Neisseria gonorrhoeae isolates and international reference strains, including those with high-level antimicrobial resistance: potential treatment option for gonorrhea? Antimicrob Agents Chemother. 2012; 56(5):2739-42.

45. Silva O, Ferreira E, Vaz Pato M, Caniça M, Gomes ET. In vitro anti-Neisseria gonorrhoeae activity of Terminalia macroptera leaves. FEMS Microbiol Lett. 2002; 217(2):271-4. 